\title{
Transversalis fascia plane block, a novel ultrasound-guided abdominal wall nerve block
}

\author{
Peter D. Hebbard, FANZCA
}

Received: 17 March 2009/Accepted: 22 April 2009/Published online: 4 June 2009

(C) Canadian Anesthesiologists' Society 2009

\section{To the Editor,}

The lateral cutaneous branches (LCB) of the thoracoabdominal nerves (T6 to L1) arise proximal to the angle of the rib, run a short distance with the main nerve, and emerge obliquely through the overlying muscles in the midaxillary line. ${ }^{1}$ They pass superficially to supply the skin of the lateral thorax, the abdomen, the iliac crest, and the upper thigh as far as the greater trochanter of the femur. As previously described, it is rare to produce block of the LCB of the subcostal (T12) and iliohypogastric (L1) nerves when performing ultrasound-guided posterior transversus abdominis plane (TAP) block. ${ }^{2}$ The subcostal and iliohypogastric nerves normally send out their LCB preceding entry or very proximal in the TAP. The LCB leave the TAP in a more posterior position than the local anesthetic of the ultrasound-guided posterior TAP block, which appears on imaging as being restricted from spreading posterior to the extent of the muscle belly. The subcostal and iliohypogastric nerves pass deep over the anterior surface of the quadratus lumborum muscle, which extends from the 12th rib to the iliac crest. The subcostal nerve then continues a short distance deep to the aponeurotic posterior extension of the transversus abdominis muscle before passing through the aponeurosis into the TAP. ${ }^{3}$ The iliohypogastric nerve continues deep to the transversus muscle aponeurosis and belly to penetrate the transversus in a more anterior and highly variable position. ${ }^{4}$

P. D. Hebbard, FANZCA ( $\square)$

Anaesthesia and Pain Management Unit,

University of Melbourne, Melbourne,

VIC, Australia

e-mail: p.hebbard@bigpond.com
Local anesthetic injected between the transversus abdominis muscle and its deep investing transversalis fascia will spread over the inner surface of the quadratus lumborum muscle and block the proximal portions of the T12 and L1 nerves. This will produce block of both the anterior and the lateral branches of these nerves. This transversalis fascia block (TFP) targets these nerves anatomically between the lumbar plexus block and the TAP block.

With the patient in a supine position, the needle is advanced from the anterior using an in-plane technique. A linear or curvilinear ultrasound probe is orientated transversely over the lateral abdomen between the iliac crest and the costal margin. The external oblique, internal oblique, and transversus abdominis muscles are imaged, and the more posterior transversus aponeurosis is distinguished. The reflection of the peritoneum curving away from the muscles from anterior to posterior is identified, and the perinephric fat, which lies behind the peritoneum and deep to the transversalis fascia, identified. The perinephric fat is generally more prominent closer to the iliac crest. The quadratus lumborum is identified medial to the aponeurosis of the transversus abdominis. It may be confused with the partly overlying erector spinae muscle, which is more superficial and often more prominent on ultrasound (Fig. 1).

To minimize the risk of peritoneal penetration or liver trauma, the block area should be sufficiently posterior so that the perinephric fat, rather than the peritoneum and liver, underlies the transversalis fascia. To enhance needle visibility, the needle insertion point is selected such that a $100-150 \mathrm{~mm}$ needle is introduced relatively perpendicular to the ultrasound beam, and the probe is slid anteriorly to image the needle throughout its course. The end point is more visible if the needle is passed through the posterior "tail" of the transversus muscle, as the transversus aponeurosis is thinner and less distinct as a separate layer. 

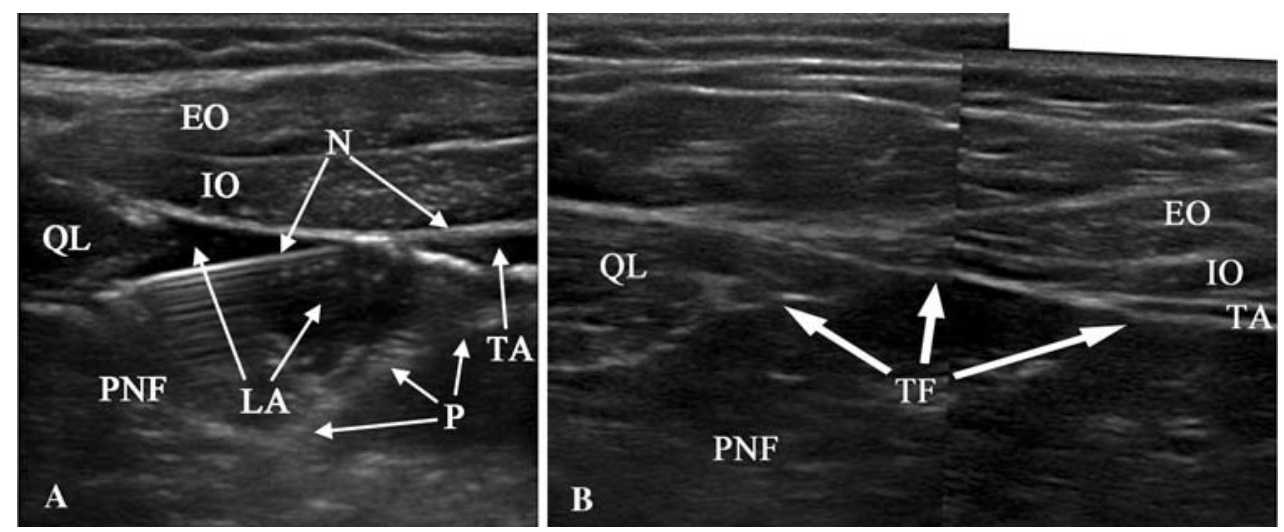

Fig. 1 Panel $A$ shows a sonogram of a completed block. The needle (N) was advanced into the hydro-dissected space and lies just anterior to the quadratus lumborum (QL). Local anesthetic (LA), peritoneum $(\mathrm{P})$, and external oblique (EO), internal oblique (IO), and transversus

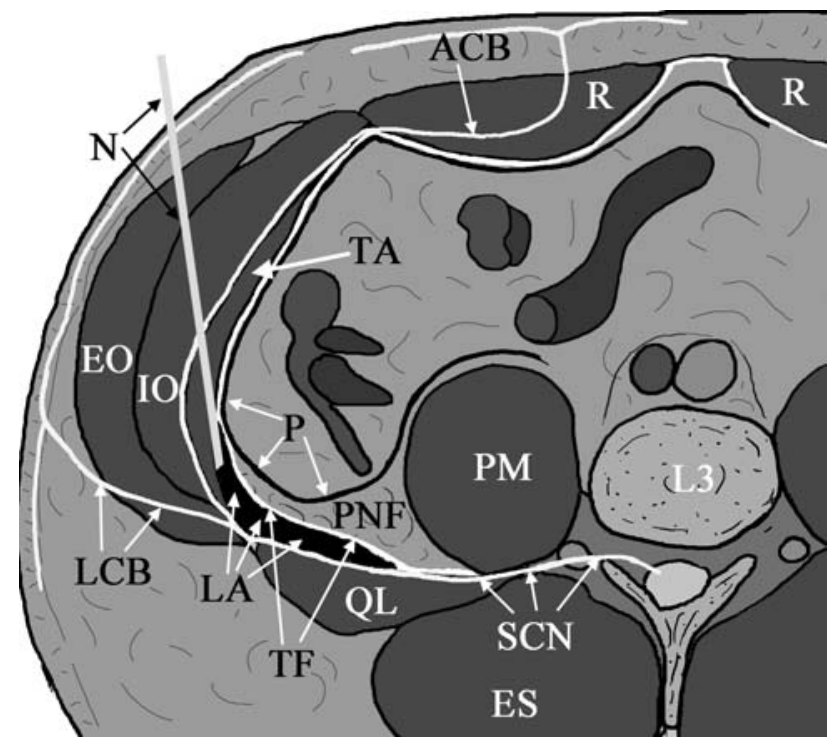

Fig. 2 Transverse diagram through the abdomen above the iliac crest. The course of the subcostal nerve ( $\mathrm{SCN})$ is indicated, including the lateral cutaneous branch (LCB) and the anterior cutaneous branch (ACB). The nerve does not actually pass along this transverse plane as it inclines downwards. The location of the local anesthetic (LA) across the anterior surface of the quadratus lumborum (QL) and behind the transversalis fascia (TF) is shown, and the needle position $(\mathrm{N})$, perinephric fat $(\mathrm{PNF})$, peritoneum $(\mathrm{P})$, and transversalis fascia (TF) are indicated. The following muscles are involved: rectus abdominis (R), erector spinae (ES), psoas (PM), transversus abdominis (TA), internal oblique (IO), and external oblique (EO)

After passing through the deep surface of transversus abdominis muscle, local anesthetic is injected to separate the transversalis fascia from the transversus muscle (Fig. 2). The local anesthetic runs both anteriorly and posteriorly. The needle can be passed posteriorly into the hydrodissected area to improve spread over the anterior surface of the quadratus lumborum. Although the needle is passed only a few millimetres beyond the TAP, the pattern of
(TA) muscles are also seen. The needle is passing through the "tail" of the transversus muscle. Panel B shows a composite sonogram of the lateral abdominal wall, including the perinephric fat (PNF) and the transversalis fascia $(\mathrm{TF})$

spread is different. Also, local anesthetic can be injected into the TAP on withdrawal to achieve a more extensive block of the anterior branches of nerves above T12.

To date, this novel TFP block has been used clinically for long-lasting analgesia in 17 patients, including five cases of iliac crest bone harvest. Other operations have included appendicectomy, cecostomy, and inguinal hernia repair, often in combination with TAP block on needle withdrawal. Early in the experience, one patient underwent a repeat block due to postoperative pain after iliac crest bone harvest, no detectable block, and a difficult block owing to the depth of imaging. The patient's pain was relieved by the second block. The other 16 patients had a detectable block to ice over the iliac crest; four of these were for bone graft harvest with excellent analgesia. There have been no complications. Compared with the more anteriorly placed ultrasound-guided posterior TAP injection, the block is limited in the anterior abdomen, as only L1, T12, and possibly T11 will be blocked. However, T12 and L1 supply laterally over the iliac crest as far as the greater trochanter enabling a simple and effective analgesic block over the iliac crest, upper lateral thigh, and lower abdomen. Also, the TFP is continuous medially with the plane of the lumbar plexus, and opening the plane with fluid may provide an alternative lateral approach to lumbar plexus block under ultrasound-guidance.

Conflicts of interest None declared.

\section{References}

1. Davies F, Gladstone RJ, Stibbe EP. The anatomy of the intercostal nerves. J Anat 1931; 66: 323-33.

2. Hebbard P, Fujiwara Y, Shibata Y, Royse C. Ultrasound-guided transversus abdominis plane (TAP) block. Anaesth Intensive Care 2007; 35: 616-7. 
3. Sakamoto H, Akita K, Sato T. An anatomical analysis of the relationships between the intercostal nerves and the thoracic and abdominal muscles in man. I. Ramification of the intercostal nerves. Acta Anat (Basel) 1996; 156: 132-42.
4. Jamieson RW, Swigart L, Anson BJ. Points of parietal perforation of the ilioinguinal and ilio-hypogastric nerves in relation to optimal sites for local anaesthesia. Q Bull Northwest Univ Med Sch. 1952; 26: 22-6. 